\title{
Use of plasma biomarkers for AT(N) classification of neurodegenerative dementias
}

Authors: Daniel Alcolea ${ }^{1,2}$, Constance Delaby ${ }^{1,3}$, Laia Muñoz ${ }^{1,2}$, Soraya Torres ${ }^{1,2}$, Teresa Estellés ${ }^{1,}$ 2, Nuole Zhu ${ }^{1,2}$, Isabel Barroeta ${ }^{1,2}$, María Carmona-Iragui ${ }^{1,2}$, Ignacio Illán-Gala ${ }^{1,2}$, Miguel Santos ${ }^{1,}$ 2, Miren Altuna ${ }^{1,2}$, Isabel Sala ${ }^{1,2}$, M. Belén Sánchez-Saudinós ${ }^{1,2}$, Laura Videla1,2 Sílvia Valldeneu $^{1,}$ ${ }^{2}$, Andrea Subirana ${ }^{1,2}$, Christophe Hirtz ${ }^{3}$, Jérôme Vialaret ${ }^{3}$, Sylvain Lehmann $^{3}$, Kaj Blennow ${ }^{4,5}$, Henrik Zetterberg ${ }^{4,5,6,7}$, Olivia Belbin ${ }^{1,2}$, Rafael Blesa ${ }^{1,2}$, Jordi Clarimón ${ }^{1,2}$, Juan Fortea ${ }^{1,2}$, Alberto Lleó $^{1,2}$

1- Sant Pau Memory Unit, Department of Neurology, Institut d'Investigacions Biomèdiques Sant Pau - Hospital de Sant Pau, Universitat Autònoma de Barcelona, Barcelona, Spain

2- Centro de Investigación Biomédica en Red en Enfermedades Neurodegenerativas, CIBERNED, Spain

3- IRMB, INM, Université de Montpellier, INSERM, CHU de Montpellier, Laboratoire de Biochimie-Protéomique clinique, Montpellier, France.

4- Department of Psychiatry and Neurochemistry, Institute of Neuroscience and Physiology, the Sahlgrenska Academy at the University of Gothenburg, Mölndal, Sweden

5- Clinical Neurochemistry Laboratory, Sahlgrenska University Hospital, Mölndal, Sweden

6- Department of Neurodegenerative Disease, UCL Institute of Neurology, London, United Kingdom

7- UK Dementia Research Institute at UCL, London, United Kingdom

\section{Corresponding authors}

Daniel Alcolea, MD, PhD. Sant Pau Memory Unit, Department of Neurology, Hospital de la Santa Creu i Sant Pau. Sant Antoni Maria Claret 167, 08025 Barcelona, Spain. Phone: +34935565986 / Fax: +34935568604 / Email: dalcolea@santpau.cat

Alberto Lleó, MD, PhD. Sant Pau Memory Unit, Department of Neurology, Hospital de la Santa Creu i Sant Pau. Sant Antoni Maria Claret 167, 08025 Barcelona, Spain. Phone: +34935565986 / Fax: +34935568604 / Email: alleo@santpau.cat

Keywords: biomarker, plasma, CSF, Alzheimer, neurodegenerative disease 


\begin{abstract}
Objectives: All categories included in the AT(N) classification can now be measured in plasma. However, their agreement with cerebrospinal fluid (CSF) markers is not fully established. A blood signature to generate the AT(N) classification would facilitate early diagnosis of patients with Alzheimer's disease (AD) through an easy and minimally invasive approach.
\end{abstract}

Methods: We measured $A \beta$, pTau181 and neurofilament light (NfL) in 150 plasma samples of the Sant Pau Initiative on Neurodegeneration cohort including patients with mild cognitive impairment, AD dementia, frontotemporal dementia, dementia with Lewy bodies and cognitively normal participants. We classified participants in the AT(N) categories according to CSF biomarkers and studied the diagnostic value of plasma biomarkers within each category individually and in combination.

Results: The plasma $A \beta$ composite, pTau181 and NfL yielded areas under the curve (AUC) of 0.75 , 0.78 and 0.88 to discriminate positive and negative participants in their respective $A, T$ and $N$ categories. The combination of all three markers did not outperform pTau181 alone (AUC=0.81) to discriminate $\mathrm{A}+\mathrm{T}+$ from $\mathrm{A}-\mathrm{T}$ - participants. There was a moderate correlation between plasma $A \beta$ composite and CSF $A \beta 1-42 / A \beta 1-40$ (Rho=-0.5, $p<0.001$ ) and between plasma $p T a u 181$ and CSF pTau181 in the entire cohort $(R h o=0.51, p<0.001)$. NfL levels in plasma showed high correlation with those in CSF (Rho $=0.78, p<0.001)$.

Conclusions: Plasma biomarkers are useful to detect the AT(N) categories, and their use can differentiate patients with pathophysiological evidence of AD. A blood AT(N) signature may facilitate early diagnosis and follow-up of patients with $A D$ through an easy and minimally invasive approach. 


\section{Background}

Cerebrospinal (CSF) amyloid $A \beta_{1-42}$, total tau (tTau), and phosphorylated tau on threonine 181 (pTau181), also named core AD biomarkers, are currently included in guidelines of the National Institute of Aging Alzheimer's Association (NIA-AA) and the International Working Group 2 to diagnose Alzheimer's disease (AD) in clinical research settings $[1,2]$. In particular, a signature of these core CSF biomarkers (increase of tTau and pTau and decrease of $A \beta_{1-42}$ or the $A \beta_{1-42} / A \beta_{1-}$ ${ }_{40}$ ratio) is currently implemented in many centres worldwide for the early diagnosis of $A D[3,4]$. Recently, a new research framework has been proposed to conceptualize the AD signature along 3 axes: $A$, reflecting amyloid pathology; $T$, reflecting tau pathology and $\mathrm{N}$, reflecting neurodegeneration. $A$ and $T$ components are more specific of the $A D$ continuum, while the $N$ component is shared with several neurodegenerative diseases.

In recent years, several plasma markers have been developed to capture pathophysiological changes observed in the central nervous system. One of the challenges of this approach is that proteins' concentrations in blood are over 100-fold lower than in CSF, and therefore their reliable measurement requires sensitive and specific methods. Different groups have reported the reliable detection of amyloid- $\beta$ biomarkers in plasma trough high-performance immune purification coupled with mass spectrometry $[5,6]$. This technology allows the prediction of brain $A \beta$ pathology and has shown a strong concordance with amyloid Positron Emission Tomography (PET) imaging and $A \beta_{1-42}$ CSF quantification, in either cognitively normal individuals, patients with mild cognitive impairment (MCl) or AD dementia [7]. Levels of Tau can also be accurately quantified in plasma using ultrasensitive immunoassays (Simoa) [8-10]. Although plasma levels of tTau performs well to identify neuronal injury in acute brain disorders (REF), it lacks disease specificity in AD [8]. In contrast, plasma levels of pTau (both pTau181 and pTau217) have proven to predict $A D$ (tau and $A \beta$ ) brain pathologies, and can differentiate $A D$ from other neurodegenerative disorders [10-12]. The same technology allows the quantification of neurofilament Light (NfL) in different biofluids, including plasma or serum, and has shown good correlation with measures in CSF [13]. NfL is a reliable measure of the $\mathrm{N}$ component and total neurodegenerative burden, and its levels in CSF increase proportionally to the degree of axonal damage [14]. Several recent studies highlight the interest of $\mathrm{NfL}$ in blood to predict disease progression and brain neurodegeneration at the pre-symptomatic stages of familial AD [13] and other neurological diseases [15]. All these blood biomarkers are highly promising as diagnostic tools for AD. However, the combination of AT(N) markers in plasma and its informativity in a cohort of patients with various neurodegenerative dementias has not been fully investigated.

In the present work, we quantified plasma levels of markers of the AT(N) categories $\left(A \beta_{42 / 40}\right.$, pTau181 and $\mathrm{NfL}$ ) in patients with different neurodegenerative dementias. We evaluated the correlation of these biomarkers with those in CSF and assessed their diagnostic accuracy alone or combined between them or with clinical or genetic data.

\section{Methods}

\section{Study participants and clinical classification}

We included 150 participants from the Sant Pau Initiative on Neurodegeneration (SPIN cohort)[16] evaluated at the Sant Pau Memory Unit (Barcelona, Spain) between November 2013 
and October 2019. We included patients with a diagnosis of Alzheimer's disease (AD), dementia with Lewy bodies (DLB), frontotemporal lobar degeneration (FTLD)-related syndromes as well as cognitively normal controls (CN) [16]. All controls had normal cognitive scores in the formal neuropsychological evaluation. All participants had received neurological and neuropsychological evaluation and provided CSF and plasma samples. A subset of participants $(n=94)$ had amyloid PET imaging with ${ }^{18} \mathrm{~F}$-florbetapir. Specific details on the SPIN cohort evaluation protocol are described elsewhere [16].

\section{CSF collection and analysis}

CSF was obtained by lumbar puncture and collected in polypropylene tubes following international recommendations [17]. Samples were processed (centrifuged $2000 \mathrm{~g}$ at $4^{\circ} \mathrm{C}$, during $10 \mathrm{~min}$ ) and aliquoted into polypropylene tubes within the first two hours after lumbar puncture. Aliquots were then stored at $-80^{\circ} \mathrm{C}$ until analysis. CSF levels of core $A D$ biomarkers $\left(A \beta_{1-42}, A \beta_{1-40}, t T a u\right.$, and pTau181) were measured in the Lumipulse fully-automated platform using commercially available kits (Fujirebio Europe, Ghent, Belgium), as previously described and according to the provider's instructions $[18,19]$.

Following the $A T(N)$ classification system [20], we used previously validated cutoffs [19] for CSF $A \beta_{1-42} / A \beta_{1-40}$ as a marker of $\beta$ amyloid deposition $(A+<0.062)$, and CSF pTau181 $(T+>63 \mathrm{pg} / \mathrm{ml})$ as a marker of Tau pathology, and we classified all participants as $A+T+, A+T-, A-T+$ or A-T-.

We used NfL in CSF as a marker of the $(\mathrm{N})$ category. Levels were measured using a commercially available ELISA kit (NFlight, UmanDiagnostics, Umeå, Sweden,) as previously described $[16,21,22]$.

\section{Blood collection and analysis}

Blood was collected in $10 \mathrm{ml}$ EDTA tubes immediately after lumbar puncture as previously described [16]. After centrifugation (2000g at 4 으, during $10 \mathrm{~min}$ ), plasma was aliquoted in polypropylene tubes and stored at -80 ㅇ $\mathrm{C}$ until analysis.

Plasma $A \beta$-peptides were quantified in Montpellier by $A \beta$ immune-purification followed by mass spectrometry analysis (IP-MS), using a protocol slightly modified from Nakamura et al. [7]. Briefly, $250 \mu \mathrm{L}$ of plasma EDTA samples were diluted and spiked with a stable-isotope-labelled (SIL) $A \beta_{1-38}$ peptide used as internal control. $A \beta$ peptides were immunoprecipitated by incubating antibody beads with the samples, followed by washing and elution of the bound peptides. The bound peptides were spotted in four replicates on Matrix-Assisted Laser Desorption Ionisation (MALDI) plate. Samples quadruplicate measurements using MALDI analysis (Shimadzu Axima) equipped with a 337-nm nitrogen laser in positive ion mode were performed using an automated acquisition method. Generated plasma biomarker profiles were processed with SPAM software (Shimadzu) to generate reports with normalized intensities using internal standard intensity (SIL-A $\beta_{1-38}$ ) and obtaining quantitative measures for $A \beta_{1-42}$ and $A \beta_{1-}$ 40. Based on the calculation of the ratios $A P P_{669-711} / A \beta_{1-42}$ and $A \beta_{1-40} / A \beta_{1-42}$, a z-score was computed for both ratios. The mean value of these two $z$-scores allows to obtain a composite biomarker score as previously described[7]. Throughout the manuscript, we used the $A \beta$ composite score as a marker of $\beta$-amyloid $(A)$ category as this is the measure that showed better accuracy in previous studies [7]. 
Plasma levels of pTau181 were quantified at the Sahlgrenska Academy at the University of Gothenburg, Möndal, Sweden, using a previously validated in-house ultrasensitive assay for the Simoa HD-X platform (Quanterix) [10].

Plasma levels of NfL were measured in Hospital Sant Pau using the ultrasensitive Single Molecule Array (Simoa) SR-X equipment (Quanterix). All samples were measured in duplicates using commercially available kits (NF-light, Quanterix) and following manufacturer's instructions [23].

All analyses were performed by experienced technicians who were blind to clinical information.

\section{APOE genotyping}

DNA was extracted using standard procedures and $A P O E$ was genotyped according to previously described methods [24].

\section{Statistical analysis}

Normality of the variables was tested by Shapiro-Wilk test. Non-normally distributed variables were log-transformed to achieve a normal distribution. Group comparisons were performed by an analysis of covariance (ANCOVA) adjusting by the effect of age and followed by Tukey's posthoc test corrected for multiple comparisons. Correction for heteroscedasticity was applied when necessary. We used $\mathrm{X}^{2}$ test to assess differences in categorical variables.

Bivariate associations between biomarkers were assessed by Spearman correlations. Diagnostic accuracy was assessed through receiver operating characteristic (ROC) analysis. We calculated areas under the curve (AUC) for all individual biomarkers and defined logistic regression models with backwards selection to assess the diagnostic accuracy of biomarkers combination. All tests were performed in Medcalc (medCalc ${ }^{\circledR}$ software ver 15.2.2) and R statistical software (v 3.6.2) using packages "psych" (2.0.8), "car" (3.0-3), "multcomp" (1.4-10), "ggplot2" (3.2.1) and "pROC" (1.15.3). Alpha threshold was set at 0.05 for all analysis.

\section{Ethical approval and consent to participate}

The ethics committee of Hospital Sant Pau approved all procedures included in this study following the standards for medical research in humans recommended by the Declaration of Helsinki. All participants or their legally authorised representative gave written informed consent before enrolment in the study.

\section{Results}

\section{Demographics, CSF and blood biomarkers}

We included a total of 150 participants from the SPIN cohort, comprising patients with AD $(n=27), \operatorname{LBD}(n=52), \operatorname{FTLD}(n=25)$ and $46 \mathrm{CN}$ participants. All participants were classified as $A+T+$, $A+T-, A-T+$ or $A-T-$, following the $A T(N)$ classification scheme according to their CSF levels of $A \beta_{1-}$ ${ }_{42} / A \beta_{1-40}$ and $p T a u 181$ in CSF.

Table 1 shows demographic and biomarker characteristics of the groups after their classification into the $A T(N)$ scheme. The A-T- group was younger compared to the other three groups and had higher MMSE average score than the $A+T+$ group. There were no differences in sex 
distribution between groups. As expected, there were differences in the distribution of clinical diagnosis between groups and in the proportion of $A P O E \varepsilon 4$ carriers.

Table 1: Demographics, clinical and biomarkers information across AT categories

MMSE: Mini-mental state examination; CSF: cerebrospinal fluid; SUVR: standardized uptake value ratio; CN: Cognitively normal; AD: Alzheimer's disease; DLB: dementia with Lewy bodies; FTLD: Frontotemporal lobar degeneration.

*Age-adjusted ANCOVA

\begin{tabular}{|c|c|c|c|c|c|}
\hline & A-T- & A-T+ & $A+T-$ & $A+T+$ & $p$ value \\
\hline $\mathbf{N}$ & 80 & 5 & 11 & 54 & \\
\hline Diagnosis (CN/AD/DLB/FTLD) & $41 / 0 / 22 / 17$ & $0 / 0 / 3 / 2$ & $1 / 1 / 6 / 3$ & $4 / 26 / 21 / 3$ & $<0.001$ \\
\hline Age (years) & $62.4(13.7)$ & $77.2(3.7)$ & $74.6(8)$ & $72.9(6.9)$ & $<0.001$ \\
\hline Female / Male (\%Female) & $24 / 22(52.2 \%)$ & $15 / 12(55.6 \%)$ & $24 / 28(46.2 \%)$ & $18 / 7(72 \%)$ & 0.35 \\
\hline MMSE score & $27.2(3.6)$ & $24(4.3)$ & $25.3(3.8)$ & $24.1(4.2)$ & $<0.001$ \\
\hline $\begin{array}{c}\text { APOE\&4- / APOE\&4+ } \\
\text { (\%APOE\&4+) }\end{array}$ & $63 / 16(20.3 \%)$ & $\begin{array}{l}5 / 0 \\
(0 \%)\end{array}$ & $\begin{array}{c}7 / 4 \\
(36.4 \%)\end{array}$ & $23 / 29(55.8 \%)$ & $<0.001$ \\
\hline $\operatorname{CSF} A \beta_{1-42}(p g / m l)$ & $1151.1(446.4)$ & $1174.8(243.2)$ & $535.9(147.7)$ & $593.4(175.7)$ & $<0.001^{*}$ \\
\hline $\operatorname{CSF} A \beta_{1-40}(p g / m l)$ & $\begin{array}{l}11059.8 \\
(3795.6) \\
\end{array}$ & $\begin{array}{c}15270.4 \\
(3207) \\
\end{array}$ & $\begin{array}{l}10276.1 \\
(2621.5) \\
\end{array}$ & $\begin{array}{l}13656.8 \\
(3521.9) \\
\end{array}$ & $<0.001^{*}$ \\
\hline $\operatorname{CSF} A \beta_{1-42} / A \beta_{1-40}$ & $0.103(0.012)$ & $0.077(0.009)$ & $0.053(0.007)$ & $0.044(0.009)$ & $<0.001^{*}$ \\
\hline CSF tTau (pg/ml) & $0.3(112)$ & $505.4(104.1)$ & $322.9(57.4)$ & $766.6(365.6)$ & $<0.001^{*}$ \\
\hline CSF pTau181 (pg/ml) & $35.3(13.3)$ & $69.4(4.2)$ & $50.5(6.7)$ & $133.7(70.9)$ & $<0.001^{*}$ \\
\hline CSF NfL (pg/ml) & $\begin{array}{c}1279.5 \\
(1566.2) \\
\end{array}$ & $3332.6(2705)$ & $1076.4(638.4)$ & $1100.2(482.8)$ & $0.14^{*}$ \\
\hline Plasma $A \beta_{1-42} / A \beta_{1-40}$ & $0.046(0.01)$ & $0.047(0.016)$ & $0.041(0.01)$ & $0.038(0.012)$ & $0.012 *$ \\
\hline Plasma pTau181 (pg/ml) & $14.8(15.7)$ & $16.6(6.8)$ & $20.8(9.8)$ & $24.6(11.4)$ & $<0.001^{*}$ \\
\hline Plasma NfL (pg/ml) & $16.2(13.8)$ & $26(15.5)$ & $22(12.1)$ & $20.2(9)$ & $0.92^{*}$ \\
\hline $\begin{array}{c}{ }^{18} \text { F-Florbetapir quantification } \\
\text { (SUVR) }\end{array}$ & $0.9(0.1)$ & NA & $1.1(0.2)$ & $1.3(0.2)$ & $<0.001^{*}$ \\
\hline
\end{tabular}

As shown in Figure 1, after adjusting by age and multiple comparisons, the $A+T+$ group had higher $A \beta$ composite scores $(p<0.001)$ and higher levels of plasma $p$ Tau181 $(p<0.001)$ compared to the A-T-. There were no significant differences in plasma NfL levels between groups ( $p=0.92)$. Levels of plasma biomarkers across clinical diagnosis are shown in Supplementary Figure 1.

Figure 1: Plasma A $\beta$ composite score (A), pTau181 (B) and Neurofilament light levels (C) across AT(N) categories Lower and upper hinges of the boxes represent the first and third quartiles for each biomarker, respectively. Central lines in the boxes correspond to median values. The upper and lower whiskers extend to the largest and smallest 
values no further than 1.5 * IQR from each hinge, respectively. Groups were compared through ANCOVA adjusting by age and multiple comparisons. Only significant differences are displayed.

Figure 1
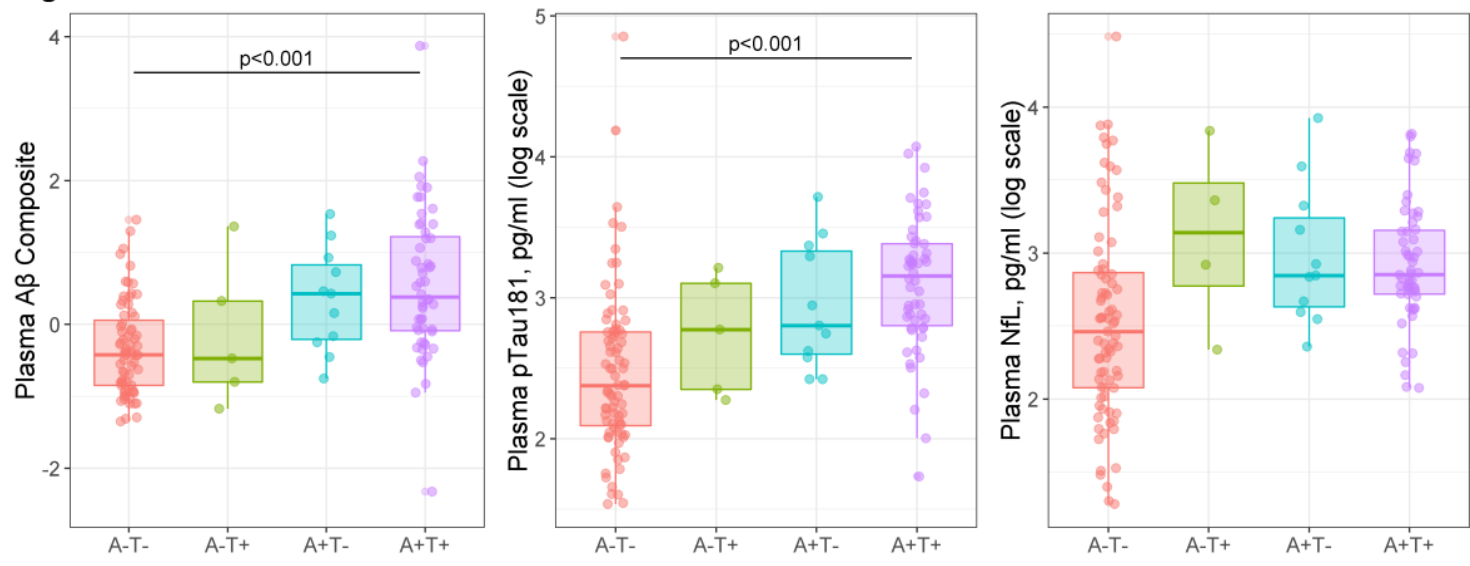

\section{Correlation of plasma biomarkers with CSF biomarkers and amyloid imaging}

We next evaluated the association between CSF and plasma biomarkers. As shown in Figure $\mathbf{2}$, the plasma $A \beta$ composite showed moderate correlation with measures in CSF in the whole sample (Rho=-0.5, $\mathrm{p}<0.001)$. Within diagnostic categories, this correlation was only significant in the DLB group ( $R h o=-0.41, p=0.003$ ). Plasma levels of $p$ Tau181 showed moderate correlation with CSF levels in the entire cohort ( $R h o=0.51, p<0.001)$. This correlation was not significant within diagnostic categories. NfL levels in plasma showed high correlation with levels in CSF (Rho $=0.78, p<0.001$ ). Within AT modalities, this correlation was significant in the groups of $\mathrm{CN}$ (Rho=0.69, $p<0.001)$, LBD (Rho=0.58, $p<0.001)$ and FTLD (Rho=0.75, $p<0.001)$.

\section{Figure 2: Correlation between AT(N) plasma biomarkers and AT(N) CSF biomarkers}

Black solid line represents the linear correlation in the whole sample. Dashed lines represent correlations within each AT category.

A

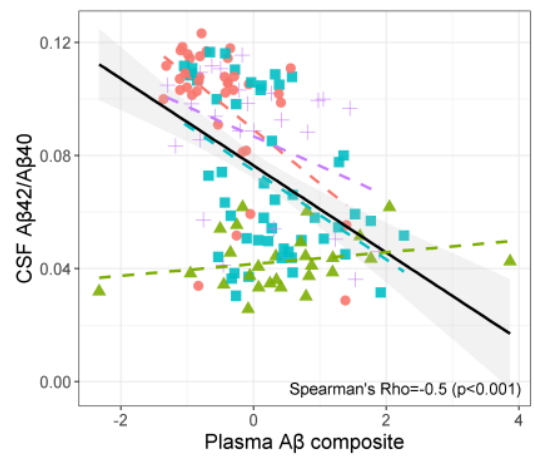

B

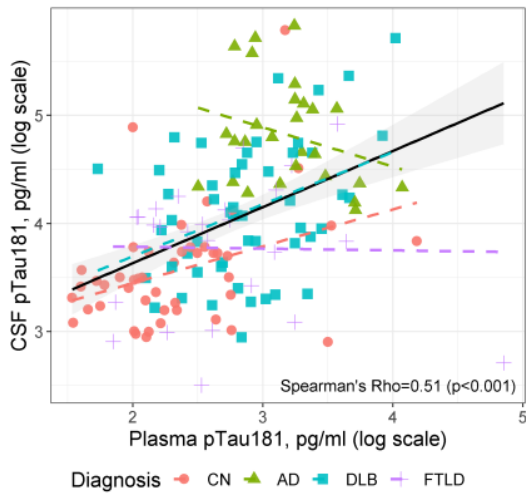

C

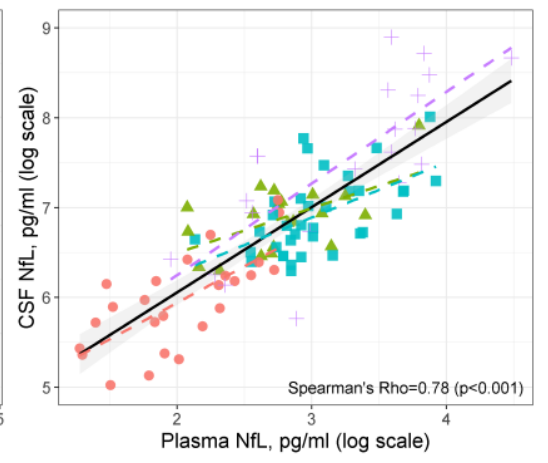

More details on bivariate correlations across and within diagnostic categories are provided as Supplementary Table 1. 


\section{Diagnostic accuracy of plasma biomarkers for the discrimination of $A+T+$ participants}

We then performed ROC analysis to assess the diagnostic accuracy of plasma biomarkers, individually and in combination, for the discrimination between participants of the $A+T+$ and the A-T- categories. This analysis would help to identify a combination of plasma biomarkers with enough diagnostic accuracy to select potential candidates for disease modifying drugs.

As shown in Figure 3, the plasma $\mathrm{A} \beta$ composite score, $\mathrm{pTau} 181$ and $\mathrm{NfL}$ levels yielded individual areas under the curve (AUC) of $0.79(95 \% \mathrm{Cl} 0.71-0.87), 0.82(95 \% \mathrm{Cl} 0.75-0.90)$ and $0.71(95 \% \mathrm{Cl}$ 0.62-0.80), respectively. In the logistic regression model with all three markers, NfL did not significantly contribute to the model, and the combination of the $A \beta$ composite score with pTau181 yielded an AUC of 0.85 (95Cl\% 0.78-0.92). This area, however, was not significantly higher than that of pTau181 alone $(p=0.65)$. The addition of age and APOE\&4 genotype to the model increased the AUC to $0.92(95 \% \mathrm{Cl} 0.87-0.97)$, which was significantly different from that of the combination of the $A \beta$ composite score with pTau181 $(p=0.04)$.

Figure 3: Receiver operating characteristic (ROC) curves for plasma biomarkers and their combination to discriminate between participants in the $\mathrm{A}+\mathrm{T}+$ and $\mathrm{A}-\mathrm{T}$ - categories.

Values between brackets did not significantly contribute to the model and were backwards-removed from the logistic model.

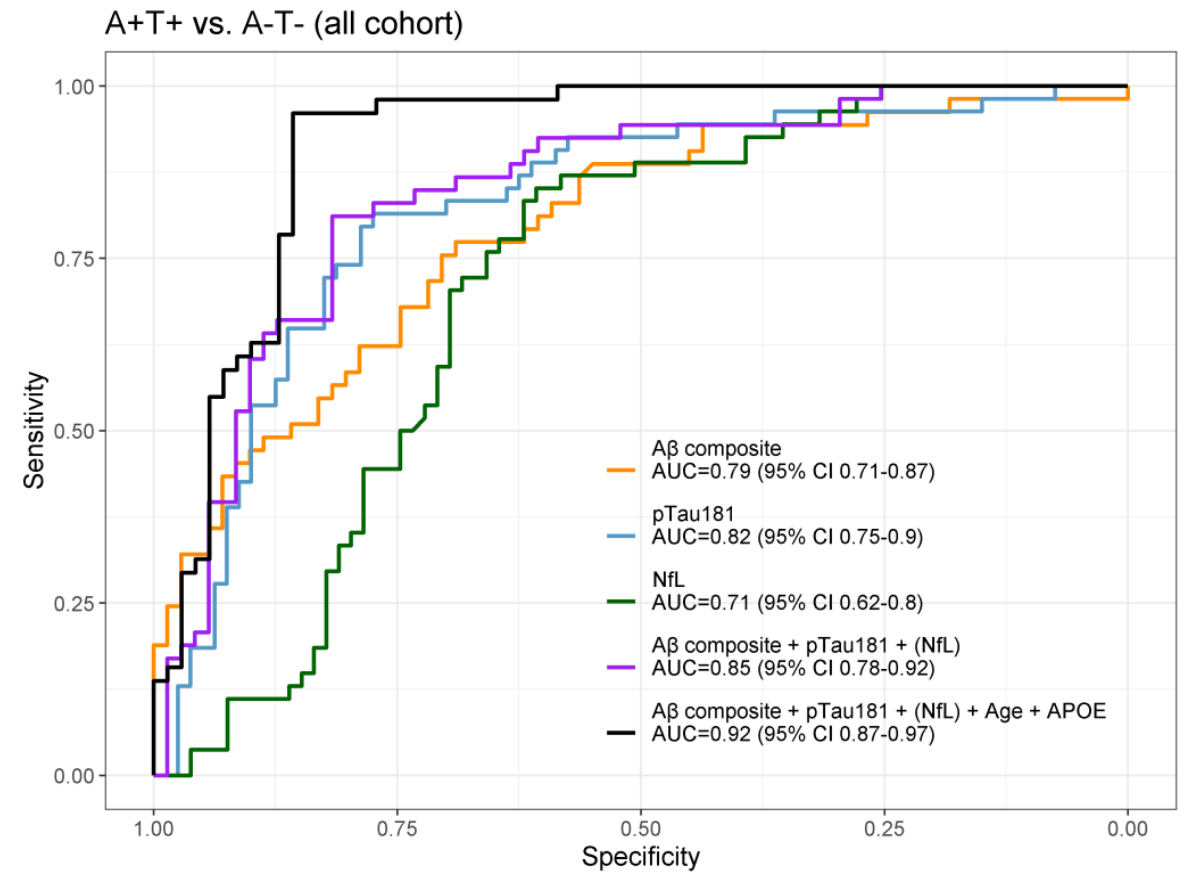

To assess the differences in the performance of plasma biomarkers between diagnostic categories, we repeated the ROC analysis separately in the subset of participants in the AD continuum (patients with $A D$ and $C N$ ) and in the subset of participants in the non-AD spectrum (DLB, FTLD and CN). Within the AD continuum, pTau181 yielded an AUC of $0.89(95 \% \mathrm{Cl} 0.81$ $0.98)$ and the combination of the three markers had an accuracy of $0.98(95 \% \mathrm{Cl} 0.96-1.0)$. The addition of age and APOE\&4 genotype did not improve the model within this group. When biomarkers were assessed in the non-AD spectrum (patients with LBD, patients with FTLD and 
controls), individual markers yielded AUC between 0.75 and 0.81 ; and the combination of all three markers had an accuracy of $0.86(95 \% \mathrm{Cl} 0.79-0.94)$ to distinguish between participants of the A+T+ category from those in the A-T- group. The inclusion of age and APOE\&4 did not significantly improve the accuracy $(p=0.23)$.

Figure 4: Receiver operating characteristic (ROC) curves for plasma biomarkers and their combination to discriminate participants in the A+T+ category from those in the A-T- category within the Alzheimer's disease spectrum (A) and in the non-Alzheimer's disease spectrum (B).

Values between brackets did not significantly contribute to the model and were backwards-removed from the logistic model.

CN: Cognitively normal; AD: Alzheimer's disease; DLB: Dementia with Lewy bodies; FTLD: Frontotemporal lobar degeneration.

A $\quad A+T+$ vs. A-T- $(A D \& C N)$

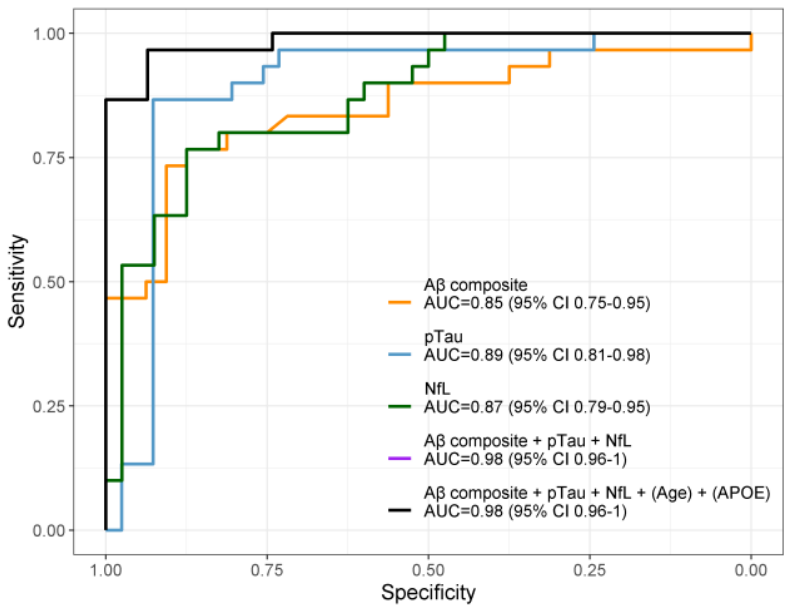

B $\quad A+T+$ vs. A-T- (DLB, FTLD \& CN)

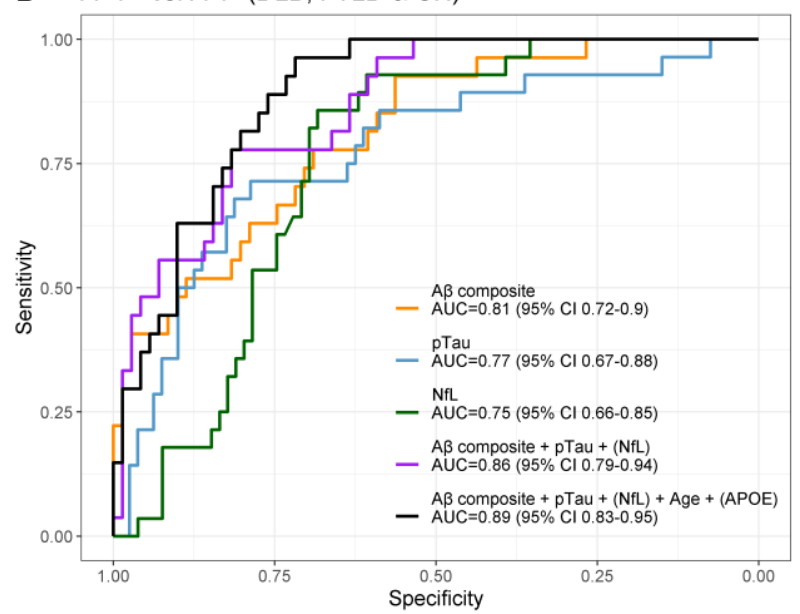

\section{Discussion}

In the present study, we describe the implementation of plasma AT(N) markers in a real-world cohort of patients with neurodegenerative dementias. We show that the combination of these biomarkers can detect the AD pathophysiology with high accuracy in a clinical setting.

$\mathrm{NfL}$ has been one of the most investigated plasma biomarkers in neurodegenerative diseases $[25,26]$. Plasma NfL levels have been shown to be useful for early detection and to track disease progression in a wide range of neurodegenerative conditions $[13,27,28]$. In previous studies, CSF and plasma levels of $\mathrm{NfL}$ correlated well with each other for all diagnoses considered $[14,29,30]$. Our results confirm increased plasma NfL levels in the different pathological conditions compared to controls, although with limited accuracy to discriminate patients A-T- and A+T+ in the whole cohort or in the non-AD spectrum when used alone ( $A \cup C \leq 0.75$ ). However, as previously described [31], plasma $\mathrm{NfL}$ has a good diagnostic performance to discriminate A-Tfrom $A+T+$ patients within the $A D$ spectrum ( $A U C=0.87$ ).

During the last decades, $A \beta$ levels have been quantified in plasma through different approaches [32], but their clinical utility has remained uncertain. However, in the last years, the development of IP-MS has contributed notable advances in the measure of $A \beta$ in blood, and despite showing limited correlation with CSF $A \beta_{1-42}$ levels, it has demonstrated high analytical 
sensitivity to predict brain $A \beta$ burden by amyloid PET [7]. In the present study, the plasma $A \beta$ composite score and plasma $A \beta_{1-42} / A \beta_{1-40}$ ratio showed significant correlations with both CSF $A \beta_{1-42} / A \beta_{1-40}$ and with amyloid PET quantification. In addition, the plasma amyloid composite biomarker was increased in $\mathrm{A}+\mathrm{T}+$ group compared to $\mathrm{A}-\mathrm{T}$ - patients in the whole cohort and had acceptable accuracy to discriminate these two subgroups ( $A \cup C=0.79$ ). This composite amyloid biomarker was the single plasma marker that best discriminated between subgroups in the non$A D$ spectrum ( $A U C=0.81$ ). It is noteworthy that IP-MS technology remains so far not easily accessible compared to other approaches, such as immunoassays (Simoa). Although highly informative, this could limit the routine use of the $A \beta$ composite score as a plasma biomarker in AD.

In this study, we also measured plasma pTau181 levels as a marker of Tau pathology. Plasma and CSF levels of pTau181 correlated well with each other in our cohort. When used alone, plasma pTau181 was the most accurate marker to discriminate A-T- and A+T+ groups in the whole cohort $(A \cup C=0.82$ ) and within the $A D$ spectrum ( $A \cup C=0.89$ ). These findings are in agreement with recently published studies $[10,25]$. In the future, it would be worth comparing the performance of this marker to that of other phosphorylated forms of Tau, such as pTau217, which has recently shown very high diagnostic accuracy in $A D$ [33].

We finally tested various combinations of these plasma markers together with $A P O E \varepsilon 4$ genotype and age, to investigate additional improvement in sensitivity and specificity. In the whole cohort, the association of the plasma $A \beta$ composite score, $\mathrm{NfL}$ and pTau181, together with age and APOE\&4 provided the best diagnostic accuracy (higher than that of pTau181 alone) with an AUC of 0.92 to discriminate $A+T+$ from $A-T$ - participants. Within the $A D$ continuum, the combination of these three plasma markers provided an accuracy of 0.98 , and the addition of age and APOE\&4 did not increase this value. This result suggests that, when there are no specific clinical features of other neurodegenerative dementias different from $A D$, the combination of plasma markers would have an extremely high accuracy to confirm the presence or the absence of an underlying $A D$ pathophysiology. On the other hand, in the non-AD spectrum, the combination of all three markers discriminated $\mathrm{A}-\mathrm{T}$ - from $\mathrm{A}+\mathrm{T}+$ with an accuracy of 0.86 without significant improvement after adding age and $A P O E \& 4$, indicating that this combination of plasma markers is also quite accurate to detect $A D$ copathology.

A strength of this study is the quantification of all AT(N) plasma and CSF biomarkers in a well characterized real-world cohort $[16,22,34]$, with the same preanalytical conditions in all participants $[18,35,36]$. The inclusion of participants with a variety of neurodegenerative dementias allowed testing the accuracy of plasma markers to detect the AD pathophysiology in different clinical scenarios. But our study has also some limitations. First, the size of some of the AT subgroups was small (especially in subgroups $A+T-$ and $A-T+$ ), which could limit the statistical power of the study. However, we accounted for heterogeneous variances in the analysis when necessary. Second, although diagnoses were established based on clinical criteria and CSF biomarkers, we had no neuropathological confirmation to ensure the correct classification of patients in AT subgroups. Third, the study has a cross-sectional design, and future studies are needed to determine whether longitudinal changes in plasma biomarkers might be useful to predict cognitive decline within the AD spectrum. 
In summary, we describe that a combination of plasma biomarkers, together with age and $A P O E \varepsilon 4$, can differentiate patients with pathophysiological evidence of $A D$ within the $A D$ spectrum but also in other neurodegenerative dementias. Such plasma signature may be applied in clinical routine as an early step for the diagnosis of $A D$ or to detect AD copathology, which would be helpful to select potential candidates for disease modifying therapies. 


\section{References}

[1] Albert MS, DeKosky ST, Dickson D, Dubois B, Feldman HH, Fox NC, et al. The diagnosis of mild cognitive impairment due to Alzheimer's disease: recommendations from the National Institute on Aging-Alzheimer's Association workgroups on diagnostic guidelines for Alzheimer's disease. Alzheimer's \& Dementia: The Journal of the Alzheimer's Association 2011;7:270-9. https://doi.org/10.1016/j.jalz.2011.03.008.

[2] Dubois B, Feldman HH, Jacova C, Hampel H, Molinuevo JL, Blennow K, et al. Advancing research diagnostic criteria for Alzheimer's disease: the IWG-2 criteria. The Lancet Neurology 2014;13:614-29. https://doi.org/10.1016/S1474-4422(14)70090-0.

[3] Andreasen N, Minthon L, Davidsson P, Vanmechelen E, Vanderstichele H, Winblad B, et al. Evaluation of CSF-tau and CSF-Abeta42 as diagnostic markers for Alzheimer disease in clinical practice. Arch Neurol 2001;58:373-9.

[4] Andreasen N, Sjögren M, Blennow K. CSF markers for Alzheimer's disease: total tau, phospho-tau and Abeta42. World J Biol Psychiatry 2003;4:147-55.

[5] Lin P-P, Chen W-L, Yuan F, Sheng L, Wu Y-J, Zhang W-W, et al. An UHPLC-MS/MS method for simultaneous quantification of human amyloid beta peptides $A \beta 1-38, A \beta 1-40$ and $A \beta 1-42$ in cerebrospinal fluid using micro-elution solid phase extraction. J Chromatogr $B$ Analyt Technol Biomed Life Sci 2017;1070:82-91.

https://doi.org/10.1016/j.jchromb.2017.10.047.

[6] Kellie J, Szapacs M. Quantitative immunocapture MS: current status and challenges in drug discovery. Bioanalysis 2014;6:2335-7. https://doi.org/10.4155/bio.14.178.

[7] Nakamura A, Kaneko N, Villemagne VL, Kato T, Doecke J, Doré V, et al. High performance plasma amyloid- $\beta$ biomarkers for Alzheimer's disease. Nature 2018;554:249-54. https://doi.org/10.1038/nature25456.

[8] Zetterberg H, Wilson D, Andreasson U, Minthon L, Blennow K, Randall J, et al. Plasma tau levels in Alzheimer's disease. Alzheimers Res Ther 2013;5:9. https://doi.org/10.1186/alzrt163.

[9] Li D, Mielke MM. An Update on Blood-Based Markers of Alzheimer's Disease Using the SiMoA Platform. Neurology and Therapy 2019;8:73-82. https://doi.org/10.1007/s40120019-00164-5.

[10] Karikari TK, Pascoal TA, Ashton NJ, Janelidze S, Benedet AL, Rodriguez JL, et al. Blood phosphorylated tau 181 as a biomarker for Alzheimer's disease: a diagnostic performance and prediction modelling study using data from four prospective cohorts. Lancet Neurol 2020;19:422-33. https://doi.org/10.1016/S1474-4422(20)30071-5.

[11] Barthélemy NR, Bateman RJ, Hirtz C, Marin P, Becher F, Sato C, et al. Cerebrospinal fluid phospho-tau T217 outperforms T181 as a biomarker for the differential diagnosis of Alzheimer's disease and PET amyloid-positive patient identification. Alzheimer's Research \& Therapy 2020;12:26. https://doi.org/10.1186/s13195-020-00596-4.

[12] Mielke MM, Hagen CE, Xu J, Chai X, Vemuri P, Lowe VJ, et al. Plasma phospho-tau181 increases with Alzheimer's disease clinical severity and is associated with tau- and amyloid-positron emission tomography. Alzheimer's \& Dementia: The Journal of the Alzheimer's Association 2018;14:989-97. https://doi.org/10.1016/j.jalz.2018.02.013.

[13] Preische O, Schultz SA, Apel A, Kuhle J, Kaeser SA, Barro C, et al. Serum neurofilament dynamics predicts neurodegeneration and clinical progression in presymptomatic Alzheimer's disease. Nat Med 2019;25:277-83. https://doi.org/10.1038/s41591-0180304-3.

[14] Gaetani L, Blennow K, Calabresi P, Di Filippo M, Parnetti L, Zetterberg H. Neurofilament light chain as a biomarker in neurological disorders. J Neurol Neurosurg Psychiatry 2019;90:870-81. https://doi.org/10.1136/jnnp-2018-320106. 
[15] Hansson O, Janelidze S, Hall S, Magdalinou N, Lees AJ, Andreasson U, et al. Blood-based $\mathrm{NfL}$ : A biomarker for differential diagnosis of parkinsonian disorder. Neurology 2017;88:930-7. https://doi.org/10.1212/WNL.0000000000003680.

[16] Alcolea D, Clarimón J, Carmona-Iragui M, Illán-Gala I, Morenas-Rodríguez E, Barroeta I, et al. The Sant Pau Initiative on Neurodegeneration (SPIN) cohort: A data set for biomarker discovery and validation in neurodegenerative disorders. Alzheimers Dement (N Y) 2019;5:597-609. https://doi.org/10.1016/j.trci.2019.09.005.

[17] Alcolea $D$, Martínez-Lage $P$, Izagirre $A$, Clerigué $M$, Carmona-Iragui $M$, Alvarez RM, et al. Feasibility of lumbar puncture in the study of cerebrospinal fluid biomarkers for Alzheimer's disease: a multicenter study in Spain. J Alzheimers Dis 2014;39:719-26. https://doi.org/10.3233/JAD-131334.

[18] Delaby C, Muñoz L, Torres S, Nadal A, Le Bastard N, Lehmann S, et al. Impact of CSF storage volume on the analysis of Alzheimer's disease biomarkers on an automated platform. Clin Chim Acta 2019;490:98-101. https://doi.org/10.1016/j.cca.2018.12.021.

[19] Alcolea D, Pegueroles J, Muñoz L, Camacho V, López-Mora D, Fernández-León A, et al. Agreement of amyloid PET and CSF biomarkers for Alzheimer's disease on Lumipulse. Annals of Clinical and Translational Neurology 2019;6:1815-24. https://doi.org/10.1002/acn3.50873.

[20] Jack CR, Bennett DA, Blennow K, Carrillo MC, Dunn B, Haeberlein SB, et al. NIA-AA Research Framework: Toward a biological definition of Alzheimer's disease. Alzheimers Dement 2018;14:535-62. https://doi.org/10.1016/j.jalz.2018.02.018.

[21] Fortea J, Carmona-Iragui M, Benejam B, Fernández S, Videla L, Barroeta I, et al. Plasma and CSF biomarkers for the diagnosis of Alzheimer's disease in adults with Down syndrome: a cross-sectional study. Lancet Neurol 2018;17:860-9. https://doi.org/10.1016/S1474-4422(18)30285-0.

[22] Delaby C, Alcolea D, Carmona-Iragui M, Illán-Gala I, Morenas-Rodríguez E, Barroeta I, et al. Differential levels of Neurofilament Light protein in cerebrospinal fluid in patients with a wide range of neurodegenerative disorders. Scientific Reports 2020;10. https://doi.org/10.1038/s41598-020-66090-x.

[23] Fortea J, Vilaplana E, Carmona-Iragui M, Benejam B, Videla L, Barroeta I, et al. Clinical and biomarker changes of Alzheimer's disease in adults with Down syndrome: a crosssectional study. The Lancet 2020;395:1988-97. https://doi.org/10.1016/S01406736(20)30689-9.

[24] McKeith IG, Boeve BF, Dickson DW, Halliday G, Taylor J-P, Weintraub D, et al. Diagnosis and management of dementia with Lewy bodies: Fourth consensus report of the DLB Consortium. Neurology 2017;89:88-100. https://doi.org/10.1212/WNL.0000000000004058.

[25] Janelidze S, Mattsson N, Palmqvist S, Smith R, Beach TG, Serrano GE, et al. Plasma Ptau181 in Alzheimer's disease: relationship to other biomarkers, differential diagnosis, neuropathology and longitudinal progression to Alzheimer's dementia. Nature Medicine 2020;26:379-86. https://doi.org/10.1038/s41591-020-0755-1.

[26] Raket LL, Kühnel L, Schmidt E, Blennow K, Zetterberg H, Mattsson-Carlgren N. Utility of plasma neurofilament light and total tau for clinical trials in Alzheimer's disease. Alzheimer's \& Dementia: Diagnosis, Assessment \& Disease Monitoring 2020;12. https://doi.org/10.1002/dad2.12099.

[27] Weston PSJ, Poole T, Ryan NS, Nair A, Liang Y, Macpherson K, et al. Serum neurofilament light in familial Alzheimer disease: A marker of early neurodegeneration. Neurology 2017;89:2167-75. https://doi.org/10.1212/WNL.0000000000004667.

[28] Palermo G, Mazzucchi S, Della Vecchia A, Siciliano G, Bonuccelli U, Azuar C, et al. Different Clinical Contexts of Use of Blood Neurofilament Light Chain Protein in the Spectrum of Neurodegenerative Diseases. Molecular Neurobiology 2020;57:4667-91. https://doi.org/10.1007/s12035-020-02035-9. 
[29] Forgrave LM, Ma M, Best JR, DeMarco ML. The diagnostic performance of neurofilament light chain in CSF and blood for Alzheimer's disease, frontotemporal dementia, and amyotrophic lateral sclerosis: A systematic review and meta-analysis. Alzheimer's \& Dementia: Diagnosis, Assessment \& Disease Monitoring 2019;11:730-43. https://doi.org/10.1016/j.dadm.2019.08.009.

[30] Hendricks R, Baker D, Brumm J, Davancaze T, Harp C, Herman A, et al. Establishment of neurofilament light chain Simoa assay in cerebrospinal fluid and blood. Bioanalysis 2019;11:1405-18. https://doi.org/10.4155/bio-2019-0163.

[31] Mattsson N, Andreasson U, Zetterberg H, Blennow K, Alzheimer's Disease Neuroimaging Initiative. Association of Plasma Neurofilament Light With Neurodegeneration in Patients With Alzheimer Disease. JAMA Neurol 2017;74:557-66. https://doi.org/10.1001/jamaneurol.2016.6117.

[32] Veerabhadrappa B, Delaby C, Hirtz C, Vialaret J, Alcolea D, Lleó A, et al. Detection of amyloid beta peptides in body fluids for the diagnosis of alzheimer's disease: Where do we stand? Critical Reviews in Clinical Laboratory Sciences 2020;57:99-113. https://doi.org/10.1080/10408363.2019.1678011.

[33] Palmqvist S, Janelidze S, Quiroz YT, Zetterberg H, Lopera F, Stomrud E, et al. Discriminative Accuracy of Plasma Phospho-tau217 for Alzheimer Disease vs Other Neurodegenerative Disorders. JAMA 2020. https://doi.org/10.1001/jama.2020.12134.

[34] Morenas-Rodríguez E, Alcolea D, Suárez-Calvet M, Muñoz-Llahuna L, Vilaplana E, Sala I, et al. Different pattern of CSF glial markers between dementia with Lewy bodies and Alzheimer's disease. Sci Rep 2019;9:7803. https://doi.org/10.1038/s41598-019-44173-8.

[35] del Campo M, Mollenhauer B, Bertolotto A, Engelborghs S, Hampel H, Simonsen AH, et al. Recommendations to standardize preanalytical confounding factors in Alzheimer's and Parkinson's disease cerebrospinal fluid biomarkers: an update. Biomarkers in Medicine 2012;6:419-30. https://doi.org/10.2217/bmm.12.46.

[36] Lehmann S, Schraen S, Quadrio I, Paquet C, Bombois S, Delaby C, et al. Impact of harmonization of collection tubes on Alzheimer's disease diagnosis. Alzheimers Dement 2014;10:S390-S394.e2. https://doi.org/10.1016/j.jalz.2013.06.008. 


\section{Acknowledgments}

We are grateful to all participants in the study and their families. We also thank all the clinical team members that were involved in the selection and assessment of participants in the SPIN cohort, and the laboratory teams in Barcelona, Montpellier and Mölndal for sample handling, biomarker analyses, and structural support.

\section{Additional information (conflicts, funding sources):}

\section{Funding sources}

This study was supported by the Fondo de Investigaciones Sanitario (FIS), Instituto de Salud Carlos III (PI14/01126 and PI17/01019 to JF, PI13/01532 and PI16/01825 to RB, PI18/00335 to MCI, PI18/00435 and INT19/00016 to DA, PI15/01618 to R.R., PI14/1561 and PI17/01896 to A.L) and the CIBERNED program (Program 1, Alzheimer Disease to Alberto Lleó), jointly funded by Fondo Europeo de Desarrollo Regional, Unión Europea, "Una manera de hacer Europa”.

This work was also supported by Generalitat de Catalunya (2017-SGR-547, SLT006/17/125 to D.A., SLT006/17/119 to J.F., SLT002/16/408 to A.L) and "Marató TV3" foundation grants 20141210 to J.F., 044412 to R.B. and 20142610 to A.L.

KB is supported by the Swedish Research Council (\#2017-00915), the Alzheimer Drug Discovery Foundation (ADDF), USA (\#RDAPB-201809-2016615), the Swedish Alzheimer Foundation (\#AF742881), Hjärnfonden, Sweden (\#FO2017-0243), the Swedish state under the agreement between the Swedish government and the County Councils, the ALF-agreement (\#ALFGBG715986), and European Union Joint Program for Neurodegenerative Disorders (JPND2019-466236).

$\mathrm{HZ}$ is a Wallenberg Scholar supported by grants from the Swedish Research Council (\#201802532), the European Research Council (\#681712), Swedish State Support for Clinical Research (\#ALFGBG-720931), the Alzheimer Drug Discovery Foundation (ADDF), USA (\#201809-2016862), the European Union's Horizon 2020 research and innovation programme under the Marie Skłodowska-Curie grant agreement No 860197 (MIRIADE), and the UK Dementia Research Institute at UCL.

[Add Additional Funding here]

The sponsors of the study did not take part in the design and conduct of the study; collection, management, analysis and interpretation of the data; writing and review of the report; or the decision to submit the article for publication.

\section{Declarations of interest}

Daniel Alcolea is employed by Hospital de la Santa Creu i Sant Pau and received research grants from Pla Estratègic de Recerca i Innovació en Salut (PERIS SLT006/17/125), and from Instituto de Salud Carlos III (PI18/00435). He participated in advisory boards from Fujirebio-Europe and received speaker honoraria from Fujirebio-Europe and from Krka Farmacéutica S.L. 
Constance Delaby is employed by Université de Montpellier. Declarations of interest: none

Laia Muñoz is employed by Biomedical Research Institute Sant Pau. Declarations of interest: none

Soraya Torres is employed by Biomedical Research Institute Sant Pau. Declarations of interest: none

Teresa Estellés is employed by Biomedical Research Institute Sant Pau. Declarations of interest: Dr. Estellés is funded by a "Río Hortega" research grant from the Institute of Health Carlos III. Nuole Zhu is employed by Hospital de la Santa Creu i Sant Pau. Declarations of interest: none Isabel Barroeta is employed by Hospital de la Santa Creu i Sant Pau. Declarations of interest: none

María Carmona-Iragui is employed by Hospital de la Santa Creu i Sant Pau. Declarations of interest: none

Ignacio Illán-Gala is employed by Hospital de la Santa Creu i Sant Pau. Declarations of interest: none

Miguel Santos is employed by Hospital de la Santa Creu i Sant Pau. Declarations of interest: He is funded by a "Juan Rodés" research grant from the Institute of Health Carlos III.

Miren Altuna is employed by Biomedical Research Institute Sant Pau. Declarations of interest: Dr. Altuna is funded by a "Río Hortega" research grant from the Institute of Health Carlos III.

Isabel Sala is employed by Hospital de la Santa Creu i Sant Pau. Declarations of interest: none

M. Belén Sánchez-Saudinós is employed by Biomedical Research Institute Sant Pau. Declarations of interest: none

Laura Videla is employed by Fundació Catalana Síndrome de Down. Declarations of interest: none

Sílvia Valldeneu is employed by Biomedical Research Institute Sant Pau. Declarations of interest: none

Andrea Subirana is employed by Biomedical Research Institute Sant Pau. Declarations of interest: none

Christophe Hirtz is employed by ... Declarations of interest: ...

Jérôme Vialaret is employed by ... Declarations of interest: ...

Sylvain Lehmann is employed by ... Declarations of interest: ...

Kaj Blennow is employed by Gothenburg University and Sahlgrenska University Hospital. ... Declarations of interest: KB has served as a consultant, at advisory boards, or at data monitoring committees for Abcam, Axon, Biogen, JOMDD/Shimadzu. Julius Clinical, Lilly, MagQu, Novartis, Roche Diagnostics, and Siemens Healthineers, and is a co-founder of Brain Biomarker Solutions in Gothenburg AB (BBS), which is a part of the GU Ventures Incubator Program.

Henrik Zetterberg is employed by the University of Gothenburg, Sahlgrenska University Hospital and University College London. Declarations of interest: $\mathrm{HZ}$ has served at scientific advisory boards for Denali, Roche Diagnostics, Wave, Samumed, Siemens Healthineers, Pinteon 
Therapeutics and $\operatorname{CogRx}$, has given lectures in symposia sponsored by Fujirebio, Alzecure and Biogen, and is a co-founder of Brain Biomarker Solutions in Gothenburg AB (BBS), which is a part of the GU Ventures Incubator Program.

Olivia Belbin is employed by Biomedical Research Institute Sant Pau. Declarations of interest: Dr. Belbin is funded by a "Miguel Servet" research grant from the Institute of Health Carlos III.

Rafael Blesa is employed by Hospital de la Santa Creu i Sant Pau and received research grants from Institute of Health Carlos III, Fundació Bancària Obra Social La Caixa and Fundació La Marató de TV3. He participated in advisory boards from Lilly and Nutricia, and he received speaker honoraria and travel funding from Novartis and Nutricia.

Jordi Clarimón is employed by Biomedical Research Institute Sant Pau and received research grants from Generalitat de Catalunya and from Institute of Health Carlos III. Declarations of interest: none

Juan Fortea is employed by Hospital de la Santa Creu i Sant Pau and received research grants from Institute of Health Carlos III, Fundació La Marató de TV3, and Pla Estratègic de Recerca i Innovació en Salut (PERIS). Declarations of interest: none

Alberto Lleó is employed by Hospital de la Santa Creu i Sant Pau and received research grants from CIBERNED, Institute of Health Carlos III and Fundación BBVA. He participated in advisory boards from Fujirebio-Europe, Nutricia, Biogen, and received speaker honoraria from Lilly.

\section{Author contribution}

DA and $A L$ designed the study. DA, CD, LM, ST, TE, NZ, IB, MCI, IIG, MS, MA, IS, MBSS, LV, SV, AS, $\mathrm{CH}, J \mathrm{~V}, \mathrm{~KB}, \mathrm{HZ}, \mathrm{JF}$ and $\mathrm{AL}$ acquired data relevant for the study. $\mathrm{DA}, \mathrm{SL}, \mathrm{KB}, \mathrm{HZ}, J \mathrm{~J}, \mathrm{JF}, \mathrm{AL}$ contributed vital reagents/tools/patents. $\mathrm{DA}, \mathrm{MCl}, \mathrm{MS}, \mathrm{SL}, \mathrm{KB}, \mathrm{HZ}, \mathrm{OB}, \mathrm{RB}, \mathrm{JC}, \mathrm{JF}, \mathrm{AL}$ obtained funding for the study. $D A$ and $C D$ performed statistical analysis. $D A, C D, A L$ contributed in analysis and interpretation of data. DA, AL participated in study supervision or coordination. CD, $\mathrm{DA}, \mathrm{AL}$ drafted the first version of the manuscript. All authors revised the manuscript for content and provided critical feedback. 


\section{SUPPLEMENTARY DATA}

Supplementary Figure 1: Plasma A $\beta$ composite score (A), pTau181 (B) and Neurofilament light levels (C) across clinical diagnostic categories.

Lower and upper hinges of the boxes represent the first and third quartiles for each biomarker, respectively. Central lines in the boxes correspond to median values. The upper and lower whiskers extend to the largest and smallest values no further than 1.5 * IQR from each hinge, respectively. Groups were compared through ANCOVA adjusting by age and multiple comparisons. Only significant differences are shown.

CN: Cognitively normal; AD: Alzheimer's disease; DLB: dementia with Lewy bodies; FTLD: Frontotemporal lobar degeneration.

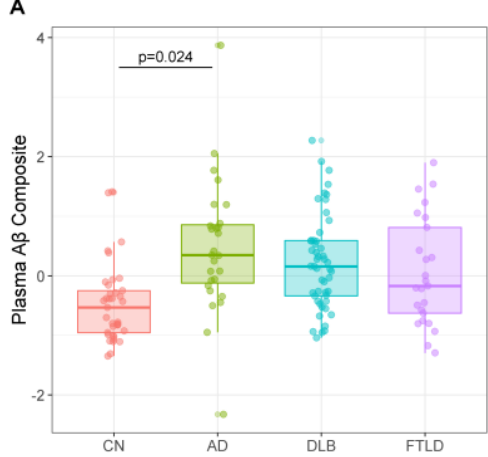

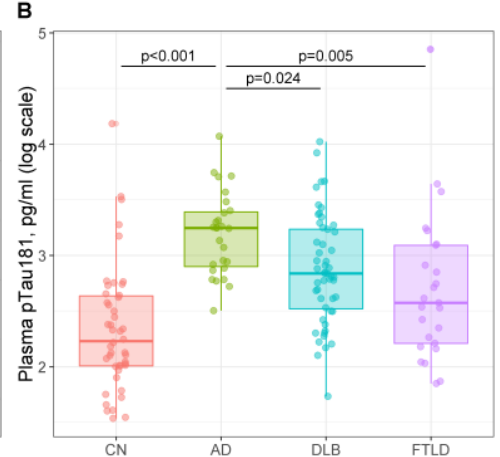

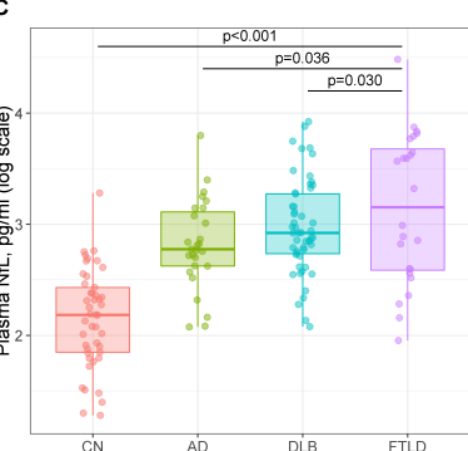

Supplementary Table 1. Spearman's correlation coefficients between plasma and other biomarkers (CSF and amyloid PET imaging)

Shaded cells indicate significant correlations after adjustment for multiple comparisons

\begin{tabular}{|c|c|c|c|c|c|}
\hline & All & $\mathrm{CN}$ & $A D$ & DLB & FTLD \\
\hline Composite - CSF A $\beta_{42 / 40}$ & $\begin{array}{l}-0.50 \\
p<0.001\end{array}$ & $\begin{array}{l}-0.42 \\
p=0.01\end{array}$ & $\begin{array}{l}0.22 \\
p=0.27\end{array}$ & $\begin{array}{l}-0.41 \\
p=0.003\end{array}$ & $\begin{array}{l}-0.33 \\
p=0.11\end{array}$ \\
\hline Plasma $A \beta_{42 / 40}-$ CSF $A \beta_{42 / 40}$ & $\begin{array}{l}0.36 \\
p<0.001\end{array}$ & $\begin{array}{l}0.30 \\
p=0.07\end{array}$ & $\begin{array}{l}-0.22 \\
p=0.28\end{array}$ & $\begin{array}{l}0.31 \\
p=0.026\end{array}$ & $\begin{array}{l}0.11 \\
p=0.60\end{array}$ \\
\hline Plasma pTau - CSF pTau & $\begin{array}{l}0.49 \\
p<0.001\end{array}$ & $\begin{array}{l}0.34 \\
p=0.04\end{array}$ & $\begin{array}{l}-0.25 \\
p=0.21\end{array}$ & $\begin{array}{l}0.30 \\
p=0.035\end{array}$ & $\begin{array}{l}0.10 \\
p=0.64\end{array}$ \\
\hline Plasma NfL - CSF NfL & $\begin{array}{l}0.77 \\
p<0.001\end{array}$ & $\begin{array}{l}0.69 \\
p<0.001\end{array}$ & $\begin{array}{l}0.41 \\
p=0.07\end{array}$ & $\begin{array}{l}0.58 \\
p<0.001\end{array}$ & $\begin{array}{l}0.75 \\
p<0.001\end{array}$ \\
\hline $\begin{array}{l}\text { Composite - Amyloid PET } \\
\text { (SUVR) }\end{array}$ & $\begin{array}{l}0.16 \\
p=0.32\end{array}$ & $\begin{array}{l}0.50 \\
p=0.39\end{array}$ & $\begin{array}{l}0.16 \\
p=0.53\end{array}$ & $\begin{array}{l}0.28 \\
p=0.32\end{array}$ & $\begin{array}{l}0.20 \\
p=0.80\end{array}$ \\
\hline $\begin{array}{l}\text { Plasma } A \beta_{42 / 40}-\text { Amyloid } \\
\text { PET (SUVR) }\end{array}$ & $\begin{array}{l}-0.14 \\
p=0.39\end{array}$ & $\begin{array}{l}-0.60 \\
p=0.28\end{array}$ & $\begin{array}{l}-0.16 \\
p=0.53\end{array}$ & $\begin{array}{l}-0.38 \\
p=0.18\end{array}$ & $\begin{array}{l}-0.20 \\
p=0.80\end{array}$ \\
\hline $\begin{array}{l}\text { Plasma pTau - Amyloid PET } \\
\text { (SUVR) }\end{array}$ & $\begin{array}{l}0.37 \\
p=0.019\end{array}$ & $\begin{array}{l}0.30 \\
p=0.62\end{array}$ & $\begin{array}{l}-0.31 \\
p=0.21\end{array}$ & $\begin{array}{l}0.29 \\
p=0.31\end{array}$ & $\begin{array}{l}1.00 \\
<0.001\end{array}$ \\
\hline $\begin{array}{l}\text { Plasma NfL - Amyloid PET } \\
\text { (SUVR) }\end{array}$ & $\begin{array}{l}0.12 \\
p=0.46\end{array}$ & $\begin{array}{l}0.90 \\
p=0.037\end{array}$ & $\begin{array}{l}-0.12 \\
p=0.65\end{array}$ & $\begin{array}{l}0.60 \\
p=0.025\end{array}$ & $\begin{array}{l}-0.40 \\
0.60\end{array}$ \\
\hline
\end{tabular}

